\title{
Ultrasound versus manipulation to determine an intercostal space for single-port thoracoscopy surgery: A Diagnostic Accuracy Study
}

chenxi li

Henan Provincial People's Hospital

Jianjun Wang

Henan Provincial People's Hospital

Zeheng Ma

Henan Provincial People's Hospital

Bing Li

Henan Provincial People's Hospital

Kang Kang

Henan Provincial People's Hospital

Li Wei

Henan Provincial People's Hospital

Wei Zhang ( $\nabla$ myhope2005@163.com )

\section{Research article}

Keywords: Intercostal space; Positioning; Ultrasound; Manipulation;

Posted Date: November 14th, 2019

DOI: https://doi.org/10.21203/rs.2.17369/v1

License: (c) (1) This work is licensed under a Creative Commons Attribution 4.0 International License.

Read Full License

Version of Record: A version of this preprint was published at World Journal of Surgical Oncology on May 23rd, 2020. See the published version at https://doi.org/10.1186/s12957-020-01870-3. 


\section{Abstract}

Background:Malposition of the intercostal space used for single-port thoracoscopy surgery can lead to severe problems for patients under general anesthesia. The focus of this study was to assess the accuracy of point-of-care ultrasound in verifying the correct position of the intercostal space used for single-port thoracoscopy surgery. Methods: A total of 200 patientsaged 18 to 65 years and classified as ASA status I- $\$ who underwent selective single-port thoracoscopic lobectomy were enrolled. After the induction of anesthesia, the patients were placed in a lateral position. An independent thoracic team left the operating room after confirming the incision position. First, the intercostal space was located by a young resident thoracic surgeon (working experience less than 1 year),andthe young thoracic surgeon left the operating room after he or she determined the location. Second, the intercostal space was located by a highly experienced thoracic surgeon (working experience more than 20 years) by manipulation; afterwards, the highly experienced thoracic surgeon left the operating room.Finally, the operation was started by a fixed independent thoracic team. After the chest cavity was opened, the final investigator verified the location of the intercostal space under direct vision through thoracoscopy. The timerequired by ultrasound and manipulation were also recorded. Results: There wereno differences between the manipulation and ultrasound methods in terms of accuracy, which was $87.2 \%(95 \% \mathrm{Cl} 0.871-0.873)$ and $85.6 \%(95 \%$ Cl0.854-0.857),respectively $(p>0.05)$. The inter-relationships between ultrasound and the standard method and between manipulation and the standard method were consistent $(p<0.05)$. Manipulation positioning showed a sensitivity of $90.6 \%$ (95\% Cl0.862-0.950) and specificity of 30\%(95\% Cl0.016-0.584), while ultrasound positioning showed a sensitivity of $87.1 \%(95 \% \mathrm{Cl} 0.820-0.921)$ and specificity of $60 \%(95 \% \mathrm{Cl} 0.296-0.904)$.Compared with that of manipulation positioning, the specificity of ultrasound positioning was higher $(p<0.05)$.No differences inPPV and NPV were found between manipulation and ultrasound positioning ( $p>0.05)$. The timerequired by ultrasound $(30.37 \pm 17.01 \mathrm{~s})$ was significantly shorter than that required by manipulation $(41.31 \pm 35.42 \mathrm{~s})(\mathrm{p}<0.05)$. Conclusions: Compared with the manipulation method, the ultrasound-guided methodcould accurately locate the intercostal space for single-port thoracoscopy surgery.Ultrasound requires less time than manipulation. Trial registration:ISRCTN10722758. Registered 04 June 2019 Keywords $\$ Intercostal space; Positioning; Ultrasound; Manipulation;

\section{Background}

Single-incision or single-port procedures, such as lobectomy, characterized by less pain and shorter length of hospital stay, have gained increasing attention with the development of endoscopic devices and techniques $[1,2]$. Since all the instruments are placed in a single small port, the accuracy of locating the intercostal space plays a very important role in surgery, proper positioning of this port is essential[3].The corresponding incision location was selected for different lesion sites[4].Malposition of the intercostal space could lead to the following problems: increased difficulty of the surgical operation, prolonged operation time, and even the requirement of another new port. 
The accuracy of traditional manipulation positioning relies on the rich clinical experience of the surgeon. Considering the anatomical variations, gender and body mass indexes of patients, manipulation positioning tends to be subjective and uncertain. With the recent popularization of point-of-care ultrasound, an increasing number of clinical practices have been increasingly dependent on ultrasound[5,6].The accuracy of locating the intercostal space relies on the effective positioning of the ribs. The ribs needed to be clearly felt by the hand to accurately locate the intercostal space in traditional manipulation positioning. In some cases, however, the ribs could not be identified clearly. In contrast, the ribs can be clearly and easily shown with ultrasound: the cortex of the rib is strongly echogenic, and the posterior periosteum is hypoechoic[7]. The ribs can be displayed in real time under ultrasound, thus avoiding subjectivity and empiricism[8].

Whether ultrasound can be used to locate intercostal spaces is unclear. The purpose of this study was to evaluate and compare the accuracy of ultrasonography and manipulation to locate an intercostal space for single-port thoracoscopic surgery.

\section{Methods}

\section{Participants}

This study was approved by the Medical Ethics Committee of Henan Provincial People's Hospital. Written informed consent and information release approvals were obtained from all patients prior to their participation in the study. The clinical trial registration code was ISRCTN10722758. The study protocol complied with the 1975 Declaration of Helsinki.

This study was performed at the Henan Provincial People's Hospital. After gaining approval, 200 subjects were enrolled in this study from June 2019 to September 2019. The inclusion criteria are as follows: aged 18 to 65 years, elective single-port thoracoscopic lobectomy, and willingness to participate. The exclusion criteria are as follows: a history of chest wounds or infections, subcutaneous emphysema, and changed surgical schedule.

\section{Study Protocol}

Overview To guarantee the blinding principle of this study, 4 researchers were included in the study. One was the primary anesthesiologist (PR), who was one of the attending faculty members of the department, was responsible for patient care throughout the study and was able to view all of the patients' vital signs and terminate the study protocol if the patient showed any signs of instability (no such events were reported). The second was a young thoracic surgeon (working experience less than 1 year) who performed the ultrasound examination (UR). The third was a highly experienced thoracic surgeon (working experience more than 20 years) who was in charge of locating the intercostal space through traditional manipulation methods (MR). The fourth was the final investigator (IR) who was in charge of verifying the intercostal space under direct vision through thoracoscopy. 
After written consent was obtained, anesthesia was inducted for all patients. All subjects were monitored by electrocardiography, pulse oximetry, invasive blood pressure recordings and bispectral index values. For fluid supplementation, $1-3 \mathrm{ml} / \mathrm{kg} / \mathrm{h}$ crystalloid was administered. Midazolam $0.05 \mathrm{mg} / \mathrm{kg}$, sufentanil $0.5 \mu \mathrm{g} / \mathrm{kg}$, etomidate $0.2 \mathrm{mg} / \mathrm{kg}$ and cisatracurium $0.2 \mathrm{mg} / \mathrm{kg}$ were intravenously administered for induction, followed by mechanical ventilation under double-lumen bronchial intubation; then, the patients were placed in a lateral position for surgery.

In this study, single-port thoracoscopic lobectomy was performed. The incision port was determined by a fixed thoracic team, which was independent of this study. There was no specific requirement for how this team should determine the intercostal space required. The location of the incision was evaluated by the UR and MR. All operations were performed by the fixed thoracic surgeon team. After induction, all patients were placed in a lateral position. The location of the incision port was then evaluated by the UR, MR and IR.

The positioning route was basically the same between the MR and the UR. The main difference between the two routes was the instrument adopted; one route was based on the hand, and the other was based on ultrasound. Briefly, the UR entered the operating room and determined which intercostal space he or she wanted to judge by ultrasound. The UR left the operating room when he or she determined a location. Then, the MR entered the operating room and determined which intercostal space he or she wanted to judge by hand. After completing his evaluation, the MR left the operating room, and the operation was started by the thoracic team. The IR entered the operating room and determined which intercostal space the incision port was actually in under direct vision with a thoracoscope. Conclusions made by the IR were recognized as the gold standard.

Positioning by the UR Previous studies suggest that a minimum of 25 to 50 examinations is needed for point-of-care ultrasound training for other topics in both the emergency department and the intensive care unit. A training curriculum of point-of-care ultrasound for the young thoracic surgeon was provided before the study started, which required a minimum of 50 examinations to complete training[9]. After the incision approach was determined, the thoracic team left the operating room. The UR went into the operating room and started his evaluation. The UR performed his examination using the Philips IU-22 machine with a 12-MHz linear probe (shown in Figure 1B). First, the probe was placed at the clavicle. Scanning was performed from the midline of the clavicle in a short-axis plane. The clavicle and subclavian arteries were first displayed on the screen. The color model could be useful for confirming the subclavian artery. The first rib, adjacent to the subclavian artery, could be easily found (shown in Figure 1C). The recognition of the first rib was important. The intercostal space below the first rib is the first intercostal space. Then, the probe was positioned downward until the probe approached the location of the surgical incision. Finally, the UR made his judgement on which intercostal space the surgical incision would be made in. Then, the UR left the operating room. The time required by the UR was recorded.

Positioning by the MR After the UR left the operating room, the MR went into the operating room and started his evaluation. The manipulation method, which is based on anatomic markers, was performed 
as follows (shown in Figure 1A). The forward protrusion, where the manubrium and the mesosternum meet, was recognized as the sternal angle. The sternal angle, attached by the second rib, could be felt by hand. The intercostal space below the second rib is the second intercostal space. After the location of the second intercostal space was confirmed, the MR continued feeling the intercostal space downward until he or she approached the location of the surgical incision. Finally, the MR made his judgement on which intercostal space the surgical incision approach would be made in. Then, the MR left the operating room. The time required by the MR was recorded.

Positioning by the IR After the UR and the MR completed their evaluations, the fixed thoracic team went into the operating room and started the operation. The standard method to confirm the location of the intercostal space was defined as counting and recognizing the ribs under direct vision after the thoracic cavity was opened. The final investigator went into the operating room and started his evaluation. After the thoracoscopic lens was placed into the thoracic cavity, the final investigator counted the ribs with the help of the thoracoscope (shown in Figure 1D). After the first rib and the subclavian artery was confirmed, the final results of where the incision was made could be easily finally verified. The conclusion made by the final investigator was recognized as the gold standard.

Data Acquisition Gender, age, body mass index, and height, and time required by the UR and MR were recorded. The results provided by the IR were regarded as the gold standard. The results provided by the UR and UR were scored as "Yes" or "No".

Statistical analysis According to the results of the preliminary experiment, the detection rates for the correct intercostal space with manipulation and ultrasound were $96.6 \%$ and $89.7 \%$, respectively. The difference between the two methods was $6.9 \%$, and the inconsistency rate was $10.3 \%$. Assuming an a of 0.05 and a power value of 0.80 , the McNemar method was used to test the differences in detecting the location of the intercostal space, and the minimum sample size was estimated to be 177 patients. Considering the data loss factor, a total of 200 patients were included in this study.

The diagnostic performance was evaluated with sensitivity, specificity, positive and negative predictive value, and accuracy. All proportions were tested using a chi-square test. The confidence intervals (Cls) of the above mentioned parameters were calculated with the Pearson method. The inter-relationships between ultrasound and the gold standard and between manipulation and the gold standard were analyzed with Spearman correlation coefficients. The data were presented as means and standard deviations or medians and quartiles. SPSS 22.0 software was used for the analysis, and the statistical data were analyzed with the chi-square test; $p<0.05$ was considered statistically significant.

\section{Results}

Data were collected from 200 subjects, and 20 subjects were excluded from the study ( 2 subjects had subcutaneous emphysema, and the operative routes were changed from a single port to other approaches in the other 18 subjects). Finally, a total of 180 subjects were included in the study. The patient demographic data are summarized in table 1. 
The location of the incision was evaluated by two independent researchers (MR and UR). Final decisions were made through direct vision by $I R$, and these decisions were taken as the gold standard. The results of the two methods are shown in table 2.

There were no differences between manipulation and ultrasound-based positioning in terms of accuracy, which was $87.2 \%$ (95\% Cl $0.871-0.873)$ and $85.6 \%$ (95\% Cl 0.854-0.857), respectively ( $p>0.05)$. Further details are shown in table 3 .

Manipulation positioning showed a sensitivity of $90.6 \%$ (95\% $\mathrm{Cl} 0.862-0.950)$ and specificity of $30 \%$ (95\% $\mathrm{Cl} 0.016-0.584)$, while ultrasound positioning showed a sensitivity of $87.1 \%(95 \% \mathrm{Cl} 0.820-0.921)$ and specificity of $60 \%$ (95囚Cl $0.296-0.904)$. Compared with that of manipulation positioning, the specificity of ultrasound was higher $(p<0.05)$. No differences in PPV and NPV were found between manipulation and ultrasound positioning $(p>0.05)$. Further details are shown in table 4.

According to the consistency test, the inter-relationships between ultrasound and the standard method and between manipulation and the standard method were consistent $(p<0.05)$. The details are shown in table 5 .

The time required by UR and MR were $27 \mathrm{~s}(20 \mathrm{~s}, 33 \mathrm{~s})$ and $30 \mathrm{~s}(22 \mathrm{~s}, 44 \mathrm{~s})$, respectively. Since the data were not normally distributed, the data were analyzed by the Mann-Whitney $U$ test; the time required by ultrasound was significantly shorter than that required by manipulation $(p=0.001<0.05)$. The data are shown in figure 2.

\section{Discussion}

To the best of our knowledge, this is the first study to evaluate the accuracy of ultrasound in locating an intercostal space for single-port thoracoscopic surgery. Our study showed that there was no difference between the accuracies of manipulation and ultrasound. The subsequent consistency test showed that both manipulation and ultrasound have good consistency with the gold standard. Currently, intercostal positioning relies more on manipulation than on ultrasound. Generally, the results from high-level surgeons with more experience were more reliable. Therefore, low-level surgeons with less experience have much more to learn. However, based on our results, after a relatively short learning period with ultrasound, even a young surgeon with minimal experience could obtain comparable accuracy. The use of ultrasound can equalize the level of less experienced doctors and flatten the learning curve.

One detail that needs our consideration is that the accuracy of ultrasound was not $100 \%$. In this study, ultrasound positioning relied on the recognition of the first rib. Since the clavicle and the first rib are closely adjacent, there is a possibility that the second rib is mistaken as the first rib under ultrasound, resulting in positioning errors. However, the ultrasound-guided method still has advantages over the manipulation method, including being intuitive, objective and visual. 
Furthermore, no difference in sensitivity was found between manual localization and ultrasound localization, which was 0.906 and 0.871 , respectively. This study demonstrated that the specificity of manipulation localization was significantly lower than that of ultrasound. Manipulation localization is susceptible to operator experience and thus is subjective. Meanwhile, manipulation localization relies heavily on anatomical markers, such as the sternal angle plane, which is typically connected to the second rib, or the subscapular angle inferior scapulae, which typically points to the seventh rib. However, anatomic variations, which are objective, may definitely affect the accuracy of manipulation positioning. For example, in some cases, the sternal hilt is very long, and the sternum angle is directly connected to the third rib. There are also other methods for positioning the 12th rib. However, the 12th rib is too short to confirm in some patients or is even absent; in rare cases, some people have a 13th rib, which would definitely cause errors[10]. Obviously, the probability of errors with manipulation positioning can increase due to anatomical variations. In addition, the difficulty of manipulation might be increased by other factors, such as obesity, the female sex and fat thickness[11,12]. In contrast, anatomical markers can be easily visualized by using ultrasound. Ultrasound is characterized as an objective method. In contrast, there is a large degree of subjectivity in manipulation localization, which may be the reason why the specificity of manipulation localization is lower than that of ultrasound localization in this study.

This study showed that the time required by ultrasound was shorter than that by required manipulation. The shortened evaluating time accelerated the working efficiency in the operating room. Moreover, the variability in the time required for ultrasound-guided positioning was small, while that of manual positioning was large, indicating that the manual localization method is more susceptible to interference by operator experience and patient factors, while the ultrasound localization method was more stable.

The study has the following shortcomings:

1. There are confounding factors in this study. In clinical practice, it is often more difficult to locate the intercostal space in obese patients, women, and patients with narrow intercostal spaces. All of these confounding factors were not excluded in this study. Although the sample size was determined by previous preliminary experiments, considering the universality of the results, some special populations failed to be excluded.

2. Only subjects who underwentsingle-port thoracoscopic surgery were enrolled. Whether our conclusion is suitable for double-port or three-port thoracoscopic surgery requires further study.

3. As discussed above, several manipulation approaches could be applied to determine the location of the intercostal space. Only one approach was studied, and the differences between ultrasound and manipulation for the same surgery were compared. There is uncertainty about the comparability of other manipulation approaches and ultrasound for the same surgery.

In summary, compared with the manipulation method, the ultrasound-guided method could be accurately applied to locate the intercostal space for single-port thoracoscopy surgery. Ultrasound requires less time than manipulation. 


\section{List Of Abbreviations}

ASA: American Society of Anesthesiologists

$\mathrm{BMI}=$ body mass index

$\mathrm{ACC}=$ Accuracy

NPV=negative predictive value

PPV = positive predictive value

\section{Declarations}

Ethics approval and consent to participate

This prospective study was approved by the Ethics Committee of Henan Provincial People's Hospital and written informed consent was obtained from patients.

Consent for publication

Not applicable

Availability of data and materials

The datasets used and analyzed during the current study are available from the corresponding author on reasonable request.

Competing interests

The authors declare that they have no competing interest

Funding

This study was supported by the grants from the Henan medical science and technology project (201602227) and Henan provincial science and technology research project (2018020844). The funding body played the roles in the design of the study and collection, analysis, and interpretation of data and in writing the manuscript. 
Author's contribution

CXL: manuscript and data analysis

JJW: implementation of the study(MR)

ZHM: implementation of the study(UR)

BL: primary anesthesiologist (PR)

KK: data collection

WL: implementation of the study (IR)

WZ: design and clinical studies

All authors read and approved the final version of the manuscript

Acknowledgments

Not applicable

\section{References}

1. Gonzalez-Rivas D, Paradela M, Fieira E,et al. Single-incision video-assisted thoracoscopic lobectomy: initial results. J Thorac Cardiovasc Surg. 2012;143(3):745-747.

2. Shen $\mathrm{Y}$, Wang $\mathrm{H}$, Feng $\mathrm{M}$,et al. Single- versus multiple-port thoracoscopic lobectomy for lung cancer: a propensity-matched studydagger. Eur J Cardiothorac Surg. 2016;49 Suppl 1i48-53.

3. Gonzalez D, de la Torre M, Paradela M,et al. Video-assisted thoracic surgery lobectomy: 3-year initial experience with 200 cases. Eur J Cardiothorac Surg. 2011;40(1):e21-28.

4. Rocco G, Khalil M, Jutley R. Uniportal video-assisted thoracoscopic surgery wedge lung biopsy in the diagnosis of interstitial lung diseases. J Thorac Cardiovasc Surg. 2005;129(4):947-948.

5. Ramsingh D, Frank E, Haughton R,et al. Auscultation versus Point-of-care Ultrasound to Determine Endotracheal versus Bronchial Intubation: A Diagnostic Accuracy Study. Anesthesiology. 2016;124(5):1012-1020.

6. Moore CL, Copel JA. Point-of-care ultrasonography. N Engl J Med. 2011;364(8):749-757.

7. Kwan RO, Miraflor E, Yeung L,et al. Bedside thoracic ultrasonography of the fourth intercostal space reliably determines safe removal of tube thoracostomy after traumatic injury. J Trauma Acute Care Surg. 2012;73(6):1568-1573. 
8. Havelock T, Teoh R, Laws D,et al. Pleural procedures and thoracic ultrasound: British Thoracic Society Pleural Disease Guideline 2010. Thorax. 2010;65 Supp/ 2ii61-76.

9. Ramsingh D, Rinehart J, Kain Z,et al. Impact assessment of perioperative point-of-care ultrasound training on anesthesiology residents. Anesthesiology. 2015;123(3):670-682.

10. Aly I, Chapman JR, Oskouian RJ,et al. Lumbar ribs: a comprehensive review. Childs Nerv Syst. 2016;32(5):781-785.

11. Flegal KM, Carroll MD, Kit BK,et al. Prevalence of obesity and trends in the distribution of body mass index among US adults, 1999-2010. JAMA. 2012;307(5):491-497.

12. Bowness JS, Nicholls K, Kilgour PM, et al. Finding the fifth intercostal space for chest drain insertion: guidelines and ultrasound. Emerg Med J. 2015;32(12):951-954.

\section{Tables}

Table 1 Patient Demographics

\begin{tabular}{|c|c|}
\hline \multicolumn{2}{|c|}{ ASA class (total $n=180$ ) } \\
\hline 口 & 38 \\
\hline 口 & 125 \\
\hline 口 & 17 \\
\hline Gender $\llbracket$ male/female $\llbracket$ & $106 / 74$ \\
\hline Age \yr】 & $55 \pm 13$ \\
\hline Height $\rrbracket \mathrm{cm} \square$ & $165.3 \pm 7.2$ \\
\hline Weightıkg』 & $66.4 \pm 10.2$ \\
\hline BMI & $24.3 \pm 3.2$ \\
\hline
\end{tabular}

Data are presented as total count or mean \pm SD.

Table 2 Positioning Results: Manipulation vs. Gold standard and Ultrasound vs. Gold standard

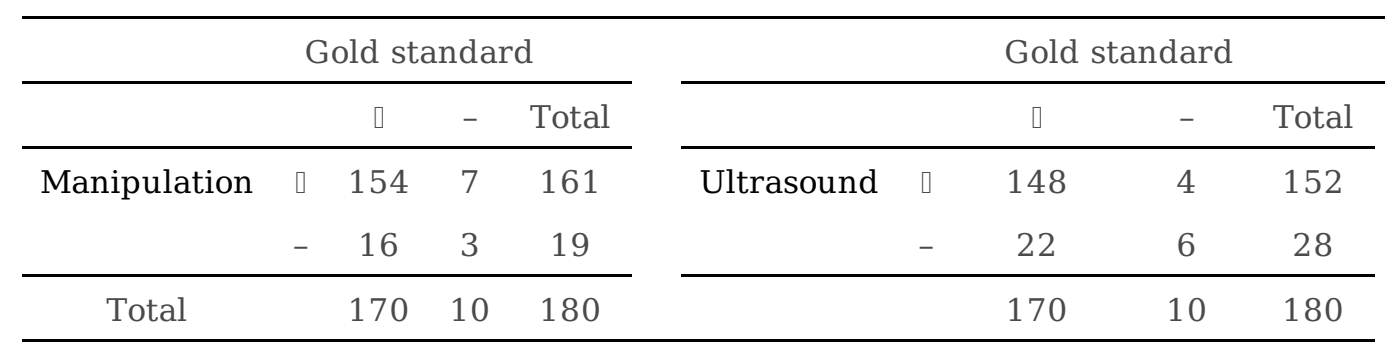

Table 3 The Accuracy of Manipulation vs. the Accuracy of Ultrasound \%(95\% CI) 


\begin{tabular}{cc}
\hline Method & Accuracy \\
\hline Ultrasound & $0.856(0.854-0.857)$ \\
Manipulation & $0.872(0.871-0.873)$ \\
$\chi^{2}$ & 0.196 \\
$p$ & 0.66 \\
\hline
\end{tabular}

Table 4 Test Characteristics of Manipulation vs. Ultrasound \%(95\% CI)

\begin{tabular}{lcccc}
\hline thod & Sensitivity & Specificity & PPV & NPV \\
\hline asound & $0.871(0.820-0.921)$ & $0.600(0.296-0.904)$ & $0.974(0.948-0.999)$ & $0.214(0.062-0.366)$ \\
pulation & $0.906(0.862-0.950)$ & $0.300(0.016-0.584)$ & $0.957(0.925-0.988)$ & $0.158(-0.006-0.322)$ \\
$\chi^{2}$ & 1.11 & 32.73 & 0.78 & 1.86 \\
$p$ & 0.29 & 0.001 & 0.38 & 0.17 \\
\hline
\end{tabular}

$\mathrm{NPV}=$ negative predictive value; PPV = positive predictive value.

Table 5 Results of the Consistency test

\begin{tabular}{ccc}
\hline Method & $p$ & Kappa value \\
\hline Ultrasound vs. Gold standard & 0.000 & 0.255 \\
\hline Manipulation vs. Gold standard & 0.039 & 0.145 \\
\hline
\end{tabular}

Figures 
A

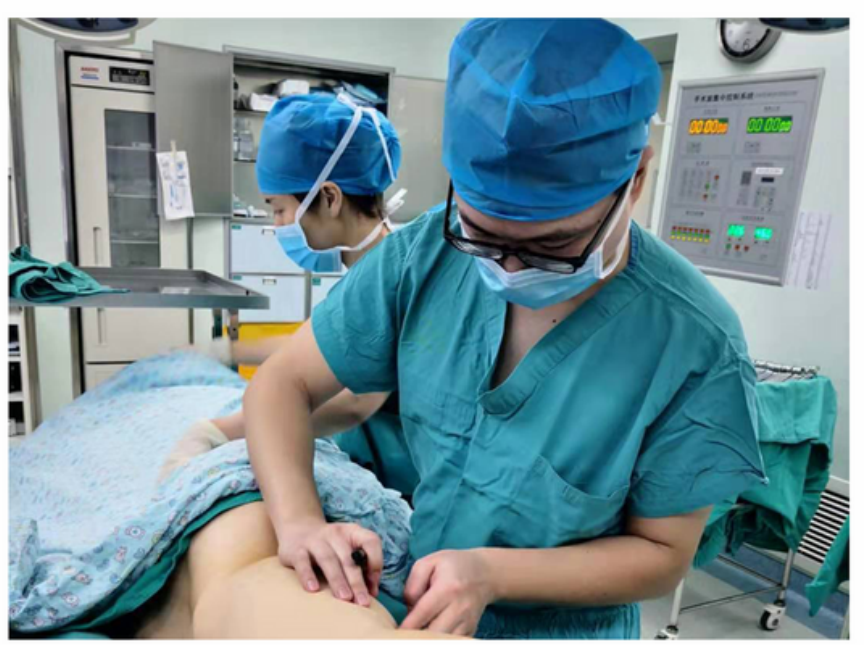

C

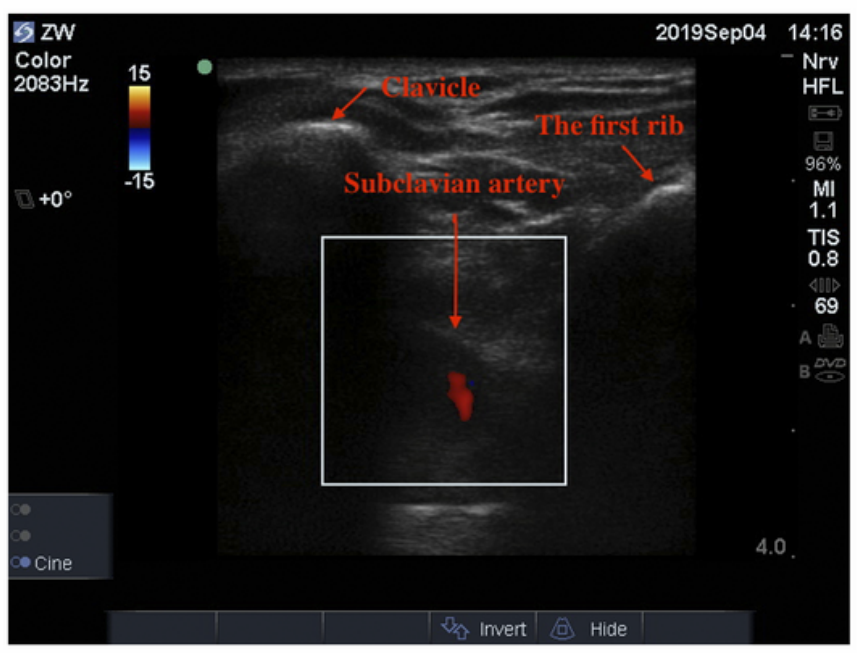

B

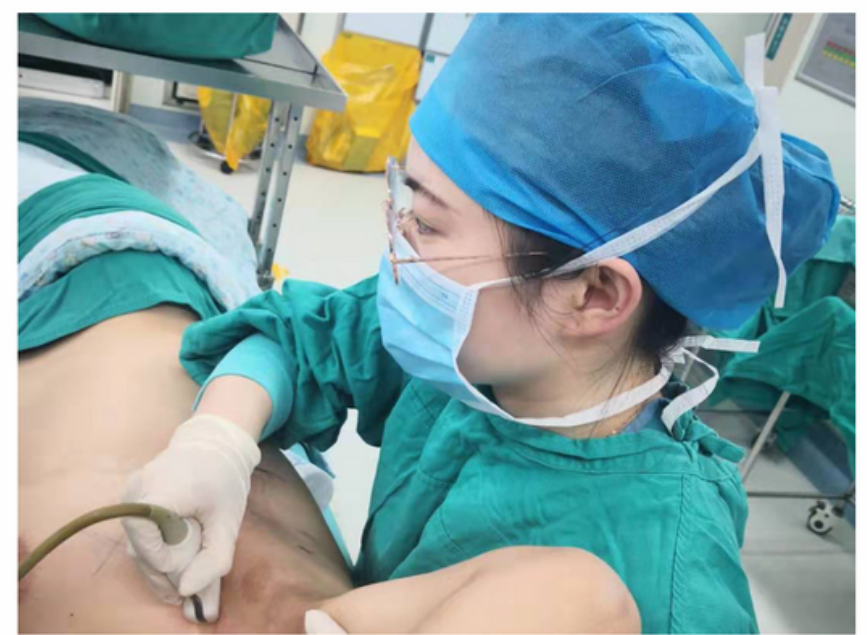

$D$

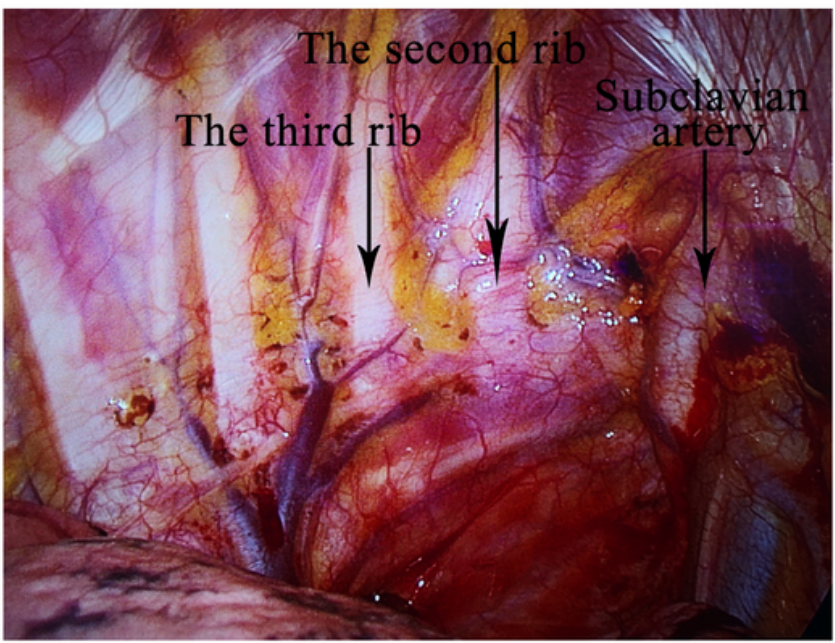

\section{Figure 1}

Title: Intraoperative illustration showing the positioning of MR, UR and IR. Intercostal space was located by manipulation (A) and ultrasound (B) respectively. As shown by the arrows, the clavicle $\llbracket$ subclavian arteries and the first rib could be easily found by ultrasound (C). The final investigator counted the ribs with the help of the thoracoscope (D). 




Figure 2

Comparison of the time required by the ultrasound and manipulation methods The time required by UR and MR were $27 \mathrm{~s}(20 \mathrm{~s}, 33 \mathrm{~s})$ and $30 \mathrm{~s}(22 \mathrm{~s}, 44 \mathrm{~s})$, respectively. Since the data were not normally distributed, the data were analyzed by the Mann-Whitney $U$ test; the time required by ultrasound was significantly shorter than that required by manipulation $(p=0.001<0.05)$.

\section{Supplementary Files}

This is a list of supplementary files associated with this preprint. Click to download.

- video.mp4 